\section{BRAZZILIAN JOURNAL \\ OF MEDICAL AND BIOLOGICAL RESHARCH}

www.bjournal.com.br
ISSN 0100-879X

Volume 42 (8) 692-775 August 2009

CLINICAL INVESTIGATION

Braz J Med Biol Res, August 2009, Volume 42(8) 756-760

The first report in Brazil of severe infection caused by community-acquired methicillin-resistant Staphylococcus aureus (CA-MRSA)

R. Rozenbaum, M.G. Sampaio, G.S. Batista, A.M. Garibaldi, G.M.F. Terra, M.J. Souza, E.N. Vieira, M.C. Silva-Carvalho, L.A. Teixeira and A.M.S. Figueiredo

The Brazilian Journal of Medical and Biological Research is partially financed by
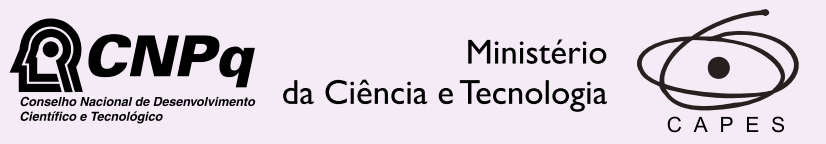

Ministério da Educação

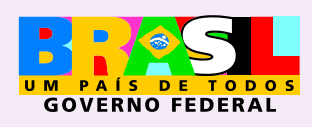

Institutional Sponsors 


\title{
The first report in Brazil of severe infection caused by community-acquired methicillin-resistant Staphylococcus aureus (CA-MRSA)
}

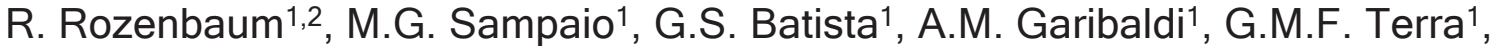 \\ M.J. Souza1 ${ }^{1}$ E.N. Vieira1', M.C. Silva-Carvalho³, L.A. Teixeira ${ }^{4}$ and A.M.S. Figueiredo ${ }^{3}$ \\ ${ }^{1}$ Hospital dos Servidores do Estado do Rio de Janeiro, Rio de Janeiro, RJ, Brasil \\ ${ }^{2}$ Hospital Samaritano, Rio de Janeiro, RJ, Brasil \\ ${ }^{3}$ Instituto de Microbiologia Prof. Paulo de Góes, Universidade Federal do Rio de Janeiro, Rio de Janeiro, \\ RJ, Brasil \\ ${ }^{4}$ Faculdade de Farmácia, Universidade Federal Fluminense, Niterói, Rio de Janeiro, RJ, Brasil \\ Correspondence to: A.M.S. Figueiredo, Instituto de Microbiologia Prof. Paulo de Góes, UFRJ, CCS, \\ Bloco I, Rua Carlos Chagas Filho, 373, 21941-590 Rio de Janeiro, RJ, Brasil. Email: agnes@micro.ufrj.br
}

Community-acquired methicillin-resistant Staphylococcus aureus (CA-MRSA) is an emergent pathogen in Brazil. However, there are no data on the prevalence of CA-MRSA. We report here the first well-characterized case of severe life-threatening CAMRSA infection in a child living in Rio de Janeiro city. The patient had many complications including hematogenous osteomyelitis and involvement of multiple sites requiring drainage of soft-tissue abscess, and pleural and pericardial empyema. The MRSA isolates recovered were genotyped using PFGE, SCCmec typing and multilocus sequence typing. Disk diffusion tests were performed following Clinical and Laboratory Standards Institute recommendations. In addition, the presence of Panton-Valentine leukocidin (PVL) was assessed by PCR amplification, using specific primers for lukF-pv (encoding for the $\mathrm{F}$ subunit of the PVL). The bacterial isolates were related to the ST30-SCCmecIV lineage (Oceania Southwest Pacific clone), a PVL producer CA-MRSA previously detected in Porto Alegre, RS, Brazil. Also, the isolates analyzed were susceptible to all non$\beta$-lactam antibiotics tested. The present report demonstrates that disseminated CA-MRSA disease is also occurring in Rio de Janeiro. Thus, the empirical treatment of moderate or severe infections suspected of being associated with CA-MRSA needs to be reviewed in order to allow prompt initiation of an effective therapy that also covers these microorganisms.

Key words: CA-MRSA; MRSA; PVL; S. aureus; Antimicrobial resistance

Research supported in part by CNPq and FAPERJ.

Received October 15, 2008. Accepted May 4, 2009

\section{Introduction}

Community-acquired infections by methicillin-resistant Staphylococcus aureus (CA-MRSA) are emerging diseases with increasing incidence worldwide (1). Most of the reports about illness caused by CA-MRSA have focused on skin and soft tissue infections, which represent the majority of infections caused by these organisms (2). Recently, CA-MRSA has also been shown to be involved in life-threatening infections. Necrotizing fasciitis, pyomyositis, septic thrombophlebitis of the extremities, rapidly progressive and necrotizing pneumonia, septic arthritis of the hips, pelvic osteomyelitis, pelvic abscesses, WaterhouseFriderichsen syndrome, and purpura fulminans with toxic shock syndrome have been reported (3), despite the fact that $S$. aureus is an unusual pathogen in some of these infectious complications, including necrotizing fasciitis and Waterhouse-Friderichsen syndrome (4). In Brazil, there 
are no data about the actual prevalence of CA-MRSA throughout the country. To our knowledge, there are only two well-documented reports describing the occurrence of mild and moderate community infections caused by CAMRSA in Rio de Janeiro, RJ and in Porto Alegre, RS $(5,6)$. The case reported here involved a 10 -year-old non-immunocompromised child living in Rio de Janeiro city, and represents the first description of a severe case of CAMRSA infection reported in Brazil. Therefore, physicians should be aware of CA-MRSA as the possible cause of lifethreatening infections in the community.

\section{Patient and Methods}

\section{Case description}

A 10-year-old non-immunocompromised girl presenting clinical manifestations of septic shock was admitted to the pediatric intensive care unit (PICU) of a general hospital located in Rio de Janeiro city on January 5, 2007. Within $48 \mathrm{~h}$ before admission, she had been assisted in the emergency department with a severe infection requiring empirical parenteral antibiotic therapy (oxacillin, ampicillin and gentamicin), pressor therapy and blood transfusion. She had no history of previous hospitalization or healthcare-associated procedures in the last year.

Characterization of the bacterial strain

$S$. aureus was identified by routine tests. Staphylococcal Cassette Chromosome mec (SCCmec) typing was carried out by multiplex PCR (7). For additional molecular characterization, genomic DNA was cut with the restriction enzyme Smal and the fragments were separated by pulsed-field gel electrophoresis (PFGE) as described previously (8). Finally, multilocus sequence typing was carried out (9).

\section{Antimicrobial susceptibility}

Disk diffusion tests were performed using Clinical and Laboratory Standards Institute (CLSI) recommendations (10). The following antimicrobial disks were tested: $5 \mu \mathrm{g}$ ciprofloxacin, $30 \mu \mathrm{g}$ chloramphenicol, $2 \mu \mathrm{g}$ clindamycin, 15 $\mu \mathrm{g}$ erythromycin, $10 \mu \mathrm{g}$ gentamicin, $1 \mu \mathrm{g}$ oxacillin, $5 \mu \mathrm{g}$ rifampin, $125 \mu \mathrm{g} / 23.75 \mu \mathrm{g}$ sulfamethoxazole-trimetroprim, $30 \mu \mathrm{g}$ tetracycline, and $30 \mu \mathrm{g}$ vancomycin.

\section{lukF-pv detection}

Panton-Valentine leukocidin (PVL) production was assessed by the detection of the gene encoding the LukF subunit of PVL using PCR amplification. The primers, the PCR assay and the program were based on Ribeiro et al. (5).

\section{Results}

\section{Clinical manifestations}

Two days before the beginning of the clinical symptoms, the patient had suffered a traumatic left ankle injury. The patient developed fever, pain, mainly localized in the left calf area, and impairment of gait, within a period of 48 $\mathrm{h}$ after the trauma. Seven days before admission she had developed furunculosis involving the buttocks. She had also developed cellulitis in the left thigh with spontaneous purulent drainage. In addition, edema, erythema and pain in the lower left and upper right limbs were also present. On admission to the PICU she had sepsis with hypotension characterized by a blood pressure of $87 \times 36 \mathrm{mmHg}$. Despite the fact that adequate fluid resuscitation was prescribed, vasoactive drugs were also necessary. Altered mental status and other perfusion abnormalities were present. Temperature varied between $35.7^{\circ}$ and $38^{\circ} \mathrm{C}$, and heart and respiratory rates were 120 beats/min and 60 breaths/min, respectively. Bilateral rales were noted in the left lower lobe. Additionally, impairment of oxygen saturation and acute respiratory failure required tracheal intubation, mechanical ventilation support and insertion of a chest tube to drain a right pleural empyema. Chest radiography showed bilateral pulmonary consolidations without cavitation with the presence of pleural effusions on both sides. Laboratory tests revealed leukocytosis (15,400 cells/ $\mathrm{mm}^{3}$ ) with a leftward shift in the white blood cell count, thrombocytopenia and elevation of both erythrocyte sedimentation rate and C-reactive protein. Sequential transthoracic echocardiography demonstrated effusion with a thickened pericardium. However, valve vegetations were not observed. Pericardiocentesis revealed purulent secretion and surgical drainage of the pericardium was required. Ultrasonography of the left thigh revealed diffuse edema extended to subcutaneous, fascia and muscle tissues.

On hospital day 10 , isolates of $S$. aureus were obtained from the blood, pleural fluid, pericardial fluid, and purulent discharge of a thigh abscess. The anterior nares screening for $S$. aureus was negative. Antimicrobial susceptibility testing had detected oxacillin resistance, which was confirmed by the amplification of the mecA gene. The parenteral antimicrobial therapy was switched to vancomycin (40 mg $\mathrm{kg}^{-1} \cdot \mathrm{day}^{-1}$ in 4 doses), clindamycin (30 mg $\mathrm{kg}^{-1} \cdot \mathrm{day}^{-1}$ in 3 or 4 doses) and ciprofloxacin (discontinued soon after). After 3 weeks, vancomycin was replaced with teicoplanin due to nephrotoxicity. Approximately 2 weeks after admission, nuclear medicine bone scanning with technetium-99m-diphosphonate and gallium-67 citrate, and later plain radiographs and nuclear magnetic resonance imaging showed hematogenous osteomyelitis characterized by 
subperiosteal osteolytic lesions affecting the distal right ulna and humerus, osteolytic lesions in the left fibula, a metaphyseal focus in the proximal left femur and right distal tibia, and osteolytic lesions in the left iliac crest. After completing an 8-week program with clindamycin and parenteral glycopeptides, the patient was discharged with the prescription of clindamycin, $300 \mathrm{mg}$ po q8h, with duration depending on clinical and laboratory improvement. After 3 months of follow-up, she was feeling good with a gradual decrease of erythrocyte sedimentation rate and an important improvement of the bone lesions characterized by

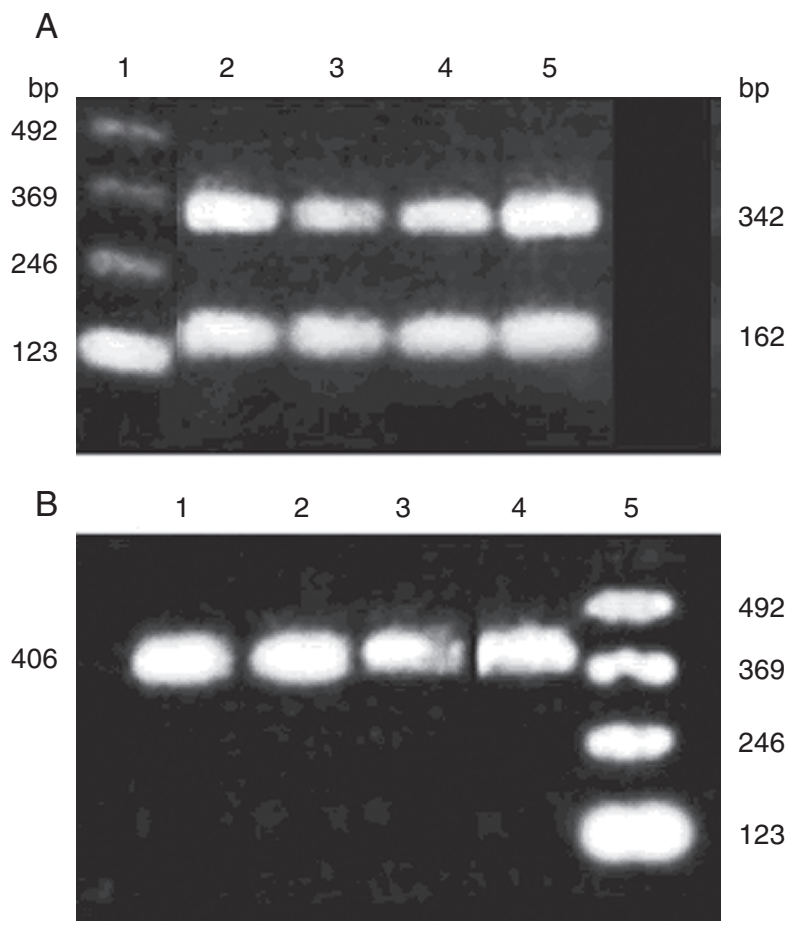

A

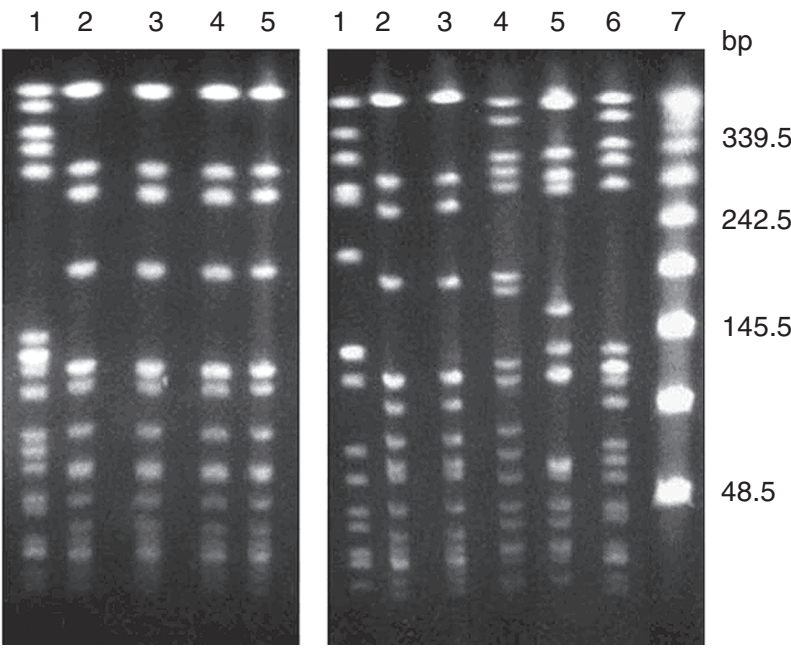

healing and subperiosteal osteogenesis.

\section{MRSA characterization}

All MRSA isolates obtained carried the SCCmec type IV element since they carried the DCS locus and no amplification was detected for the CIF and KDP loci (Figure 1A). In addition, all isolates displayed a PFGE pattern very similar (about 3-4-band difference) to that of CAMRSA representative of the ST30-SCC mec IV lineage (Figure $2 A, B$ ), previously detected in Brazil, causing skin/soft tissue infections and septic arthritis $(5,6)$. Indeed, the

Figure 1. $A$, Detection of the DCS locus and mecA by PCR. The multiplex PCR for SCCmec typing amplified only one expected band of $342 \mathrm{bp}$ for DCS-specific primers. The lower band in Figure $1 \mathrm{~A}$ is a specific fragment of the mecA gene (162 bp) obtained using previously described primers (1). Lane 1, 123-bp molecular size marker. Lanes 2-5, isolates obtained from the patient. $B$, Detection of the lukF-pv gene coding for PantonValentine leukocidin (406 bp) using specific primers (1). Lanes 14 , isolates obtained from the patient. Lane 5, 123-bp molecular size marker

Figure 2. A, Pulsed-field gel electrophoresis (PFGE) of the CAMRSA isolates obtained from different sites in the patient's body. Lane 1, BMB9393 (isolate representative of the Brazilian epidemic clone); lanes 2-5, CA-MRSA isolates of the ST30SCCmec IV lineage obtained from the patient. $B$, PFGE patterns of representatives of different MRSA lineages. Lane 1, WB78 (a representative of the ST8-SCCmecIV, USA300 clone). Lanes 2,3, WB79 and WB80 (isolates representative of the ST30SCCmec IV lineage, Oceania Southwest Pacific clone). Lane 4, WB81 (a representative of the ST1-SCCmec IV lineage, USA 400 clone). Lane 5, AM771 (a representative of the ST5SCC mec IV lineage, pediatric clone or USA800 clone). Lane 6, BMB9393 (a representative of the ST239-SCCmec III, Brazilian epidemic clone). Lane 7, Lambda ladder molecular size marker. 
isolates obtained from the child were also typed as ST30 by multilocus sequence typing.

\section{Antimicrobial susceptibility}

Although resistant to $\beta$-lactam antibiotics (oxacillin), the isolates were susceptible to all non-ß-lactam antimicrobials tested.

\section{Discussion}

CA-MRSA infections are currently emerging in Brazil, posing a challenge in terms of diagnosis and initiation of effective therapy in order to reduce morbidity and mortality. Successful management of bacteremia and other invasive infections by CA-MRSA will depend on the timely recognition of this microorganism as a cause of severe infections. Hence, the source of $S$. aureus bacteremia and the metastatic complications need to be identified in order to ensure the best treatment. The duration of antimicrobial therapy will depend on the extent of infection. Endocarditis needs to be excluded by transesophageal echocardiography. The present case had many complications including hematogenous osteomyelitis and involvement of multiple sites requiring drainage of a soft-tissue abscess and pleural and pericardial empyema. Also, long-term antibiotic therapy was necessary to guarantee successful treatment. Treatment failure and complications from hematogenous osteomyelitis have been repeatedly linked to a short duration of antimicrobial therapy (11). Therefore, we planned parenteral therapy for a minimum of $6-8$ weeks, which was completed with oral therapy with clindamycin until clinical and radiological improvement, and a return of the erythrocyte sedimentation rate to the normal range.

The treatment of serious infections by CA-MRSA deserves some considerations. Vancomycin has been the mainstay for the treatment of MRSA bacteremia. However, this drug has been associated with slower bacterial clearance and worse response rates among patients with bacteremia and endocarditis caused by methicillin-susceptible $S$. aureus (MSSA) when compared with those associated with antistaphylococcal $\beta$-lactam agents (11). In the case reported here, the CA-MRSA infection was not recognized until the 5th day after admission, when the appropriate antimicrobial therapy could be initiated. At present, in Rio de Janeiro, a possible therapeutic approach if $S$. aureus is suspected to cause life-threatening community infections would be the association of an antistaphylococcal $\beta$-lactam agent (e.g., oxacillin: $200 \mathrm{mg} \cdot \mathrm{kg}^{-1} \cdot \mathrm{day}^{-1}$ in 6 doses) and a glycopeptide (e.g., vancomycin: 40 to $60 \mathrm{mg} \cdot \mathrm{kg}^{-1}$. day $^{-1}$ in 4 doses) to maximize coverage for both MRSA and MSSA until identification of the agent and susceptibility testing. However, the benefits of this association need to be studied. Vancomycin is the glycopeptide of choice when nervous system infection and/or endocarditis are present. Otherwise, in the absence of these complications, teicoplanin, which tends to be better tolerated, can be an alternative $(3,11)$. A greater concern is the increasing identification of $S$. aureus isolates that do not respond to vancomycin therapy in vivo. Not rarely, resistance is not easily demonstrated by clinical and laboratory testing because many of the strains that fail to respond to therapy with vancomycin exhibit the heteroresistance phenomenon, which requires special antimicrobial tests for detection. One or more adjunctive agents (i.e., gentamicin, rifampin) may be added for potential synergy when treating life-threatening infections (i.e., sepsis, central nervous system infection, endocarditis) $(3,12)$.

In the case reported here, the addition of clindamycin to the vancomycin regimen was due to the presence of pneumonia with pleural empyema and the possibility of hematogenous osteomyelitis in association with septic arthritis. In this case, the isolate was susceptible to both erythromycin and clindamycin, and thus did not show inducible resistance to the latter drug. Clindamycin should not be used for empirical treatment in communities with high rates of inducible clindamycin resistance, since treatment failures may occur. Thus, in case of erythromycin resistance, the D test (13) for screening inducible resistance to clindamycin should be performed before prescribing clindamycin $(14,15)$. It was recently reported that clindamycin markedly suppressed the production of PVL, hemolysins, and toxic shock syndrome toxin 1 (16). Agents with activity against CA-MRSA available for parenteral therapy include vancomycin, teicoplanin, linezolid, daptomycin, tigecycline, and trimethoprim-sulfamethoxazole. However, no controlled trials comparing the efficacy of parenteral drugs in the treatment of serious CA-MRSA infections are available.

In Brazil, we have previously reported 18 cases of infections involving CA-MRSA isolates $(5,6)$. Two of these cases were community-acquired septic arthritis (11\%) in adult patients, and both CA-MRSA isolates belonged to the ST30-SCCmec IV lineage and were PVL producers. Except for this report, no case of osteomyelitis associated with CA-MRSA has been reported thus far in our country. Recently, another PVL-producing CA-MRSA lineage, the so called Queensland-QId lineage (ST93-SCCmec IV), was found to cause osteomyelitis in a child at a Queensland hospital, Australia. Additionally, these cases were also associated with deep venous thrombosis (17).

After the present manuscript was submitted, our group characterized two other cases of severe community infec- 
tions associated with the PVL producer ST30-SCCmec IV $S$. aureus lineage affecting children in Rio de Janeiro city.

CA-MRSA is emerging in Brazil as an agent of potentially serious infections. The most common lineage isolated thus far is related to the OSP clone (ST30-SCCmecIV), a PVL producer CA-MRSA previously detected in Australia. This lineage seems to be well adapted to cause invasive diseases in the community. $S$. aureus bacteremia is commonly associated with sepsis and other metastatic complications as the result of hematogenous dissemination. In areas where CA-MRSA infections have been recently reported, but the magnitude of the problem is unknown, we suggest that suspected life-threatening CAMRSA infections deserve prompt initiation of empirical effective combination therapy against both MRSA and MSSA, until identification and susceptibility testing are completed

\section{References}

1. Kluytmans-Vandenbergh MF, Kluytmans JA. Communityacquired methicillin-resistant Staphylococcus aureus: current perspectives. Clin Microbiol Infect 2006; 12 (Suppl 1): 9-15.

2. Davis SL, Perri MB, Donabedian SM, Manierski C, Singh A, Vager D, et al. Epidemiology and outcomes of communityassociated methicillin-resistant Staphylococcus aureus infection. J Clin Microbiol 2007; 45: 1705-1711.

3. Moellering RC Jr. Current treatment options for communityacquired methicillin-resistant Staphylococcus aureus infection. Clin Infect Dis 2008; 46: 1032-1037.

4. Gordon RJ, Lowy FD. Pathogenesis of methicillin-resistant Staphylococcus aureus infection. Clin Infect Dis 2008; 46 (Suppl 5): S350-S359.

5. Ribeiro A, Dias C, Silva-Carvalho MC, Berquo L, Ferreira FA, Santos RN, et al. First report of infection with community-acquired methicillin-resistant Staphylococcus aureus in South America. J Clin Microbiol 2005; 43: 1985-1988.

6. Ribeiro A, Coronado AZ, Silva-Carvalho MC, FerreiraCarvalho BT, Dias C, Rozenbaum R, et al. Detection and characterization of international community-acquired infections by methicillin-resistant Staphylococcus aureus clones in Rio de Janeiro and Porto Alegre cities causing both community- and hospital-associated diseases. Diagn Microbiol Infect Dis 2007; 59: 339-345.

7. Oliveira DC, de Lencastre H. Multiplex PCR strategy for rapid identification of structural types and variants of the mec element in methicillin-resistant Staphylococcus aureus. Antimicrob Agents Chemother 2002; 46: 2155-2161.

8. Soares MJ, Teixeira LA, Nunes MR, da Silva Carvalho MC, Ferreira-Carvalho BT, Figueiredo AM. Analysis of different molecular methods for typing methicillin-resistant Staphylococcus aureus isolates belonging to the Brazilian epidemic clone. J Med Microbiol 2001; 50: 732-742.

9. Multi Locus sequence typing. http://saureus.mlst.net. Accessed April 17, 2009

10. CLSI - Clinical and Laboratory Standards Institute. Perfomance standards for antimicrobial disk susceptibility tests. 9th edn. Approved Standard M2-A9. Wayne: CLSI document M100-S17; 2007.

11. Cosgrove SE, Fowler VG Jr. Management of methicillinresistant Staphylococcus aureus bacteremia. Clin Infect Dis 2008; 46 (Suppl 5): S386-S393.

12. Wootton M, Howe RA, Hillman R, Walsh TR, Bennett PM, MacGowan AP. A modified population analysis profile (PAP) method to detect hetero-resistance to vancomycin in Staphylococcus aureus in a UK hospital. J Antimicrob Chemother 2001; 47: 399-403.

13. Fiebelkorn KR, Crawford SA, McElmeel ML, Jorgensen JH. Practical disk diffusion method for detection of inducible clindamycin resistance in Staphylococcus aureus and coagulase-negative staphylococci. J Clin Microbiol 2003; 41: 4740-4744

14. Frank AL, Marcinak JF, Mangat PD, Tjhio JT, Kelkar S, Schreckenberger PC, et al. Clindamycin treatment of methicillin-resistant Staphylococcus aureus infections in children. Pediatr Infect Dis J 2002; 21: 530-534.

15. Siberry GK, Tekle T, Carroll K, Dick J. Failure of clindamycin treatment of methicillin-resistant Staphylococcus aureus expressing inducible clindamycin resistance in vitro. Clin Infect Dis 2003; 37: 1257-1260.

16. Stevens DL, Ma Y, Salmi DB, Mclndoo E, Wallace RJ, Bryant AE. Impact of antibiotics on expression of virulenceassociated exotoxin genes in methicillin-sensitive and methicillin-resistant Staphylococcus aureus. J Infect Dis 2007; 195: 202-211.

17. Nourse C, Starr M, Munckhof W. Community-acquired methicillin-resistant Staphylococcus aureus causes severe disseminated infection and deep venous thrombosis in children: literature review and recommendations for management. J Paediatr Child Health 2007; 43: 656-661. 